\title{
2019 Laos children's dental caries experience survey
}

\section{Chae-Young Rhee ${ }^{1}$, Ja-Won Cho ${ }^{1}$, Hyun-Jun Yoo ${ }^{1}$, Nitthasack Phommavongsa ${ }^{1,2}$, Yong-Su Ahn ${ }^{1}$, Hyung-Suk $\mathrm{Oh}^{1}$}

${ }^{1}$ Department of Preventive Dentistry, College of Dentistry, Dankook University, Cheonan, Republic of Korea

${ }^{2}$ Department of Pediatric Dentistry, Faculty of Dentistry, University of Health Science, Vientiane, Laos

Objectives: The aim of this study was to determine the dental caries experience of children in Laos.

Methods: Oral examinations were performed on a total of 1,540 students in 513 primary school students, 537 middle school students, and 490 high school students and the results analyzed.

Results: The dft index (decayed-filled primary teeth index) of 6-year-old primary school children was 6.04. The DMFT index (decayed-filled-missing permanent teeth index) was 1.59 in 12-year-old middle school children and 2.04 in 15-year-old middle school children.

Conclusions: Caries experience in most of the age groups was on the high side. It is considered that in Laos, a treatment project to stop the progression of caries is necessary in parallel with a prevention project to lower the caries fatality rate.

Key Words: Dental caries, Dental health surveys, Laos

Copyright (C) 2021. Korean Academy of Preventive Dentistry. All rights reserved.

This is an Open Access article distributed under the terms of the Creative Commons Attribution Non-Commercial License (http://creativecommons.org/licenses/ by-nc/4.0) which permits unrestricted non-commercial use, distribution, and reproduction in any medium, provided the original work is properly cited. 